\title{
Very High Sensitivity Laser Gyroscopes for General Relativity Tests in a Ground Laboratory
}

\author{
J. Belfi, F. Bosi, A. Di Virgilio \\ INFN Pisa, Pisa, Italy \\ Email: belfi@pi.infn.it
}

\author{
N. Beverini, G. Carelli, \\ U. Giacomelli, E. Maccioni, A. Simonelli \\ Dip. di Fisica, Università of Pisa, Pisa, Italy
}

\author{
A. Beghi, D. Cuccato, \\ A. Donazzan, G. Naletto
}

Dip. Ingegneria dell'Informazione, Università di Padova, Padua, Italy

\author{
A. Porzio \\ CNR-SPIN, and INFN Napoli, \\ Naples, Italy
}

M. G. Pelizzo

CNR-IFN and INFN Sezione di Padova, Padua ,Italy

\author{
C. Altucci, R. Velotta \\ Dip. di Fisica, Università di Napoli Federico II \\ and INFN Napoli, Naples, Italy
}

Legnaro, Padua, Italy
Legori Nazionali INFN,

\author{
A. Tartaglia \\ Dip. di Scienza Applicata e Tecnologia \\ Turin, Italy
}

\begin{abstract}
Two ring laser gyroscopes are being developed by the INFN in view of fundamental Physics applications. The scope of this activity is to 'fill the gap' between the present state of the art technology and the requirements in sensitivity and accuracy needed for general relativity tests. The first prototype, called GP2 and located at INFN Pisa, is dedicated to the interferometric control of the ring laser cavity form factor. The second prototype, called GINGERino, is a larger ring laser located deep underground (INFN LNGS) and has the scope of characterizing the low frequency rotational noise of the site. We show the most recent results of the two experiments.
\end{abstract}

\section{INTRODUCTION}

Large frame helium neon ring lasers are unique instruments for geophysics and geodesy since they monitor the instantaneous angular velocity of their reference frame with an extremely high resolution, detecting both local ground rotations and global effects. Recently the Gross Ring " $G$ " at the Wettzell Geodetic Observatory has reached the outstanding resolution on the Earth rotation rate of $3 \times 10^{-9}$ (about $0.25 \mathrm{prad} / \mathrm{s}$ with few hours of integration time [1]. This level is less than one order of magnitude far from the requirement, in term of resolution, for the direct observation of the effects induced by General Relativity on the local measurement of the Earth rotation rate [2]. The GINGER (Gyroscopes IN GEneral Relativity) project ([3],[4]) aims at developing a rotations detector capable to probe General Relativity effects on ground. We consider two independent estimates of the Earth angular velocity vector: one performed in the Earth-based laboratory reference system with a ring laser array, say it $\vec{\Omega}$, and the other performed with respect to an inertial reference frame (distant observer), say it $\vec{\Omega}^{\prime}$. According to the Einstein's Relativity predictions, to the leading order, the difference $\vec{\Omega}=\vec{\Omega}-\vec{\Omega}^{\prime}$ between the two measurements is given by:

$$
\overrightarrow{\delta \Omega} \sim \frac{G M}{c^{2} R} \Omega_{E} \sin \theta \hat{e_{\theta}}+\frac{G}{c^{2} R^{3}} J_{E}\left[\hat{j}-3 \hat{J_{E}} \cdot \hat{e_{r}}\right]
$$

where $G$ is the gravitational constant, $R$ is the Earth mean radius, $c$ is the speed of light, $\Omega_{E}$ is the Earth's angular velocity, $M$ is the Earth mass, $J_{E}$ is the Earth's angular momentum, $\theta$ is the laboratory colatitude, $\hat{j}$ is the direction of the Earth rotation vector, $\hat{e_{r}}$ and $\hat{e_{\theta}}$ are respectively the radial and the tangential directions in the local meridian plane. The first term is related to the Earth mass only and corresponds to the geodetic effect. The second term is related to the rotation of the Earth mass and corresponds to the frame dragging or Lense-Thirring effect. The order of magnitude of the different relativistic terms depends on the latitude, but always below 1 part per billion of the Earth-s rotation rate. The main issues to approach for achieving such an ambitious goal are connected to:

- $\quad$ Reduce the light shot-noise level: this can be obtained by increasing the size of the ring cavity (for a given set of mirrors the sensitivity scales as $L^{\alpha}$ with $\alpha \sim 5$ [5]).

- Improve the long-term stability: laser parameters and the environmental parameters drifts can be reduced respectively by means of active controls and by selecting a properly isolated installation. A deep underground sensor location seems necessary to reduce surface noise due to changing weather patterns and local hydrological effects.

- Correction of the nonlinear laser dynamics.Intracavity dynamics introduces nonreciprocal effects consisting in a null-shift term in the laser gyroscope response. To achieve the required accuracy, an estimate of these contributions must be implemented [6].

In the following we will describe the mechanical and optical properties of the two INFN prototypes and their recent performance.

\section{GP2 RING LASER}

Resolving the Earth rotation rate below the level of one part per billion, requires to control the ring laser geometrical 
scale factor $K_{s}$ to the same level of accuracy. Being $K_{s}=\frac{4 A}{\lambda P}$, the stabilization of the only laser cavity perimeter $P$, directly related to the ring laser optical frequency emission, is not sufficient. In fact, also the area $A$ enclosed by the beam path must be controlled. The aim is to reduce the fluctuation associated with the deformation of the optical cavity, stabilizing with a sub-nanometer accuracy the variation of the mirror interdistances.

More specifically, the beam circulating inside a cavity with four spherical mirrors has 12 degrees of freedom in space, corresponding to the 3 coordinates in space of each of the four mirrors. Tracing out the three rigid translations and rotations, we have 6 remaining degrees of freedom determining the cavity shape. Our basic idea is to constrain these degrees of freedom by exploiting the symmetry properties of a closely regular square shape. In particular, we proposed [7] an active stabilization approach. This is done by injecting the two diagonal Fabry-Pérot resonators, formed by the two couples of opposite cavity mirrors with the same ultra-stable reference laser, and correct the position of the cavity mirrors by means of piezoelectric nanometric transducers. In this way the stability of the laser wavelength is transfered to the square diagonals and the cavity is affected only to the second order by the perturbations on the mirrors positions along the residual 4 degrees of freedom. These last can be eventually optimized by controlling the cavity perimeter length. In fact, once the fixed diagonals length constraint is considered, the regular square configuration corresponds to a saddle point for the perimeter length function. The experimental setup installed at the INFN in Pisa is shown in Fig.1.

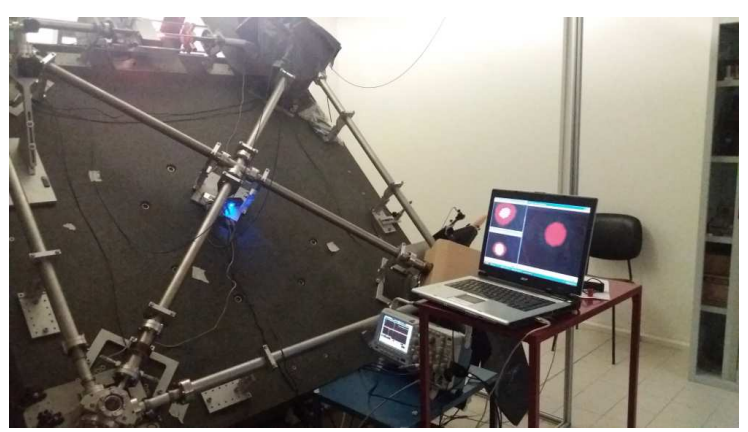

Fig. 1: GP2 ring laser is a $1.6 \times 1.6 \mathrm{~m}^{2}$ square cavity and is oriented at the maximum kinematical Sagnac signal, i.e. with the laser plane perpendicular to the Earth's rotation axis. Three webcams are used for monitoring the beam profiles of three laser beams: the two modes resonating in the diagonals and the ring laser emission itself.

The experimental technique for the diagonal cavities control is based on the multi-frequency phase modulation of a reference laser, and is described in detail in [8]. This provides the estimate of both the cavity resonance frequency and the free-spectral range of the two resonators, so that an absolute length measurement can be achieved. In Fig. 2 the optical scheme of the geometry control apparatus is reported. A subnanometer length stabilization of the diagonals cavities has been obtained with a signal integration time of few hundred seconds. The feedback signals controlling the two diagonal cavity lengths are reported in Fig. 3. Both signals follow the

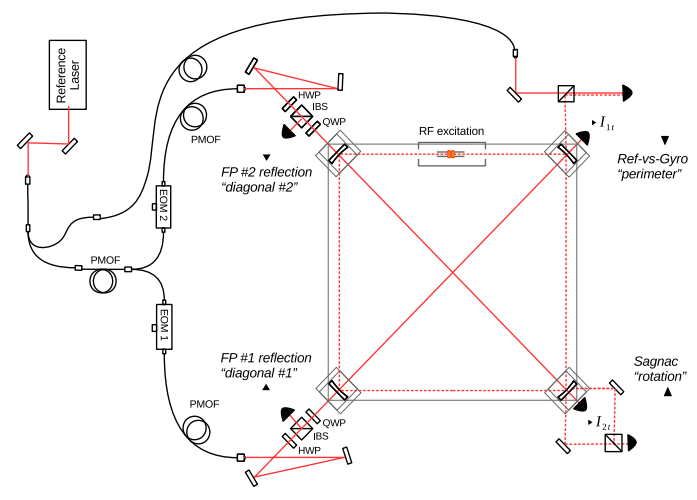

Fig. 2: Optical setup for the interrogation of the two diagonal resonators. EOM: Electro-Optic Modulator. PBS: Polarizing Beam Splitter. IBS: Intensity Beam Splitter. PMOF: Polarization Maintaining Optical Fiber. HWP: Half Wave Plate. QWP: Quarter Wave Plate. PZT: Piezoelectric Transducer. F: neutral filter. FP: Fabry Pérot cavity.
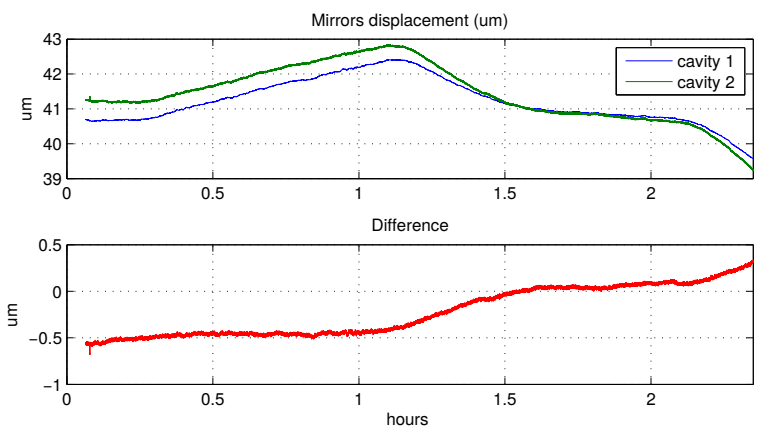

Fig. 3: Closed-loop corrections to the two couples of opposite mirrors forming the GP2 diagonal cavities.

room temperature fluctuation in the laboratory, that to date is not provided by a temperature stabilization system. The switch off of the air-conditioning/heating, is visible and clearly affect the trend of the two signals. The difference between the two signal corrections, plotted in the same graph, accounts for timedependent temperature gradients in the laboratory, differential mechanical relaxations, and also slightly different mechanical responses of the piezoelectric translator stages. The square cavity length (perimeter) is the other quantity that is estimated. Two methods provide precise cavity length measurements: the beat frequency between the ring laser and the reference laser and the ring laser longitudinal mode spacing between different longitudinal modes emitted by the ring laser. The second method requires to operate the laser in multi mode regime and provides an absolute value of the ring laser roundtrip time.

\section{GINGERINO RING LASER}

A comparison between a local measurement of the Earth's rotation rate with a measurement provided by the IERS system (based on the VLBI network) requires a strong rejection of the local rotational noise. This is mainly given by surface geo- 
physical phenomena connected to atmospheric load variations caused by changing weather patterns and local hydrology. The LNGS deep underground laboratory is a possible candidate location, being located underneath more than thousand meters of solid rock. GINGERino ring laser gyroscope is located inside the LNGS lab, in an isolated area, outside the principal experimental rooms. Its aim is to perform high resolution local measurements of the vertical component of the Earth rotation rate, and analyze the different noise contributions by comparing them with the measurement provided by seismic instrumentation installed on top of the ring laser rigid structure: one tilt-meter with nrad resolution (2-K High Resolution Tiltmeter (HRTM), Lipmann) and two high performance seismometers (Trillium 240s and Guralp CMG 3T 360s). GINGERino uses the mechanics of G-Pisa [9] which is made up of 4 mirror boxes connected by vacuum pipes. The optical resonator is a square cavity $3.6 \mathrm{~m}$ in side-length equipped with four spherical mirrors $4 \mathrm{~m}$ in radius of curvature. Each mirror box can be independently tilted with micrometric screws, so to align the optical cavity. The ring laser (Fig. 4) is tightly attached to a cross structure made of black African granite, composed by a central octagonal massive block (3 tons), and four lightened arms each weighting $\sim 800 \mathrm{~kg}$. The granite structure is screwed
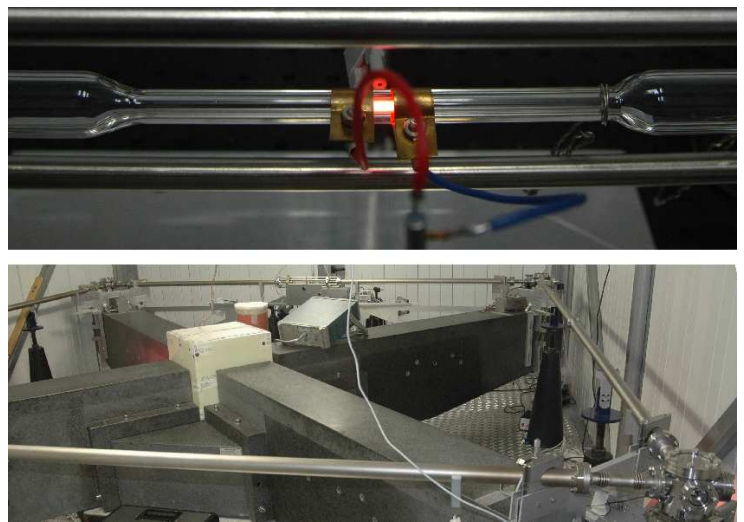

Fig. 4: Upper picture: detail of the ring laser excitation system. A radio-frequency discharge generates a $\mathrm{He}-\mathrm{Ne}$ plasma inside a pyrex tube, $4 \mathrm{~mm}$ in diameter. Lower picture: the GINGERino vacuum chamber, containing the square cavity.

to a reinforced concrete block integral to the underneath bedrock. The African black granite has been chosen because it can be machined with high precision and has a low thermal expansion coefficient $\left(7 \mathrm{ppm} /{ }^{\circ} \mathrm{C}\right)$. The installation area was at a temperature of about $8{ }^{\circ} \mathrm{C}$ with a relative humidity close to the dew point all the year round. The whole installation is now protected by a large anechoic chamber. Infrared lamps are used to increase the temperature inside the box, thus reducing the relative humidity from more than $90 \%$ down to $50-60 \%$. Typical environmental parameters trends inside the chamber are shown in Fig. 5.

The optical apparatus allowing us to run the laser continuously and observe the Earth induced Sagnac frequency is much simpler than the setup under study for the GP2 ring laser. The four cavity mirrors are spherical mirrors with 4 meters of radius of curvature. Presently, the ring down time of resonant light inside the ring is about $150 \mu \mathrm{s}$, corresponding to
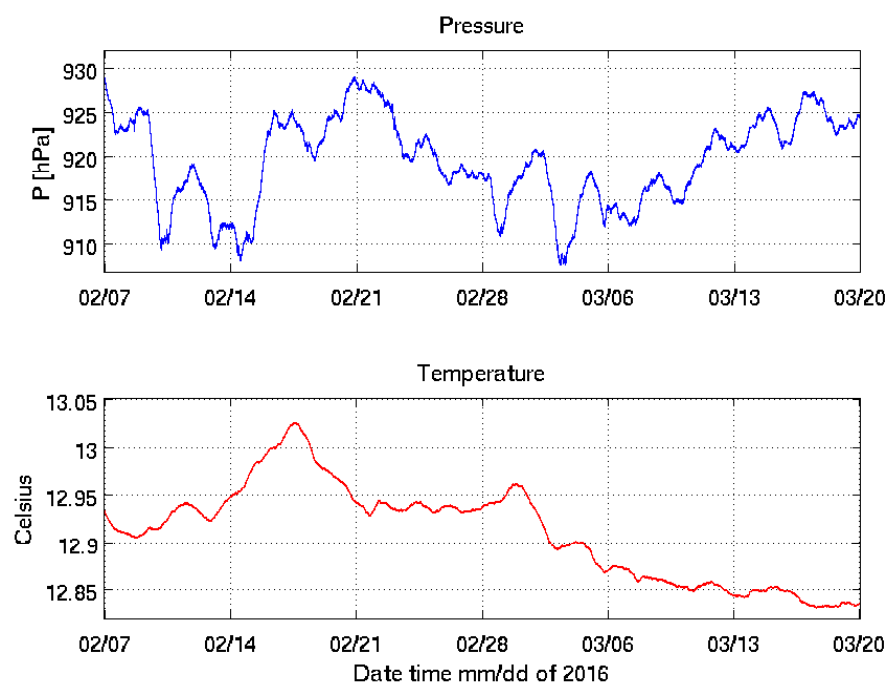

Fig. 5: Environmental parameters inside the GINGERino experimental chamber inside the Gran Sasso laboratory.

an average reflectivity of $99.98 \%$, mainly limited by scattering losses. This is definitely a low level performance for a set of supermirrors and we expected to achieve the level of $99.999 \%$ with a new set of mirrors to be installed before summer 2016.

The Sagnac frequency recorded by GINGERino running without any frequency stabilization system is shown in Fig. 6. The noise level evaluated from the power spectral density is shown in Fig.7

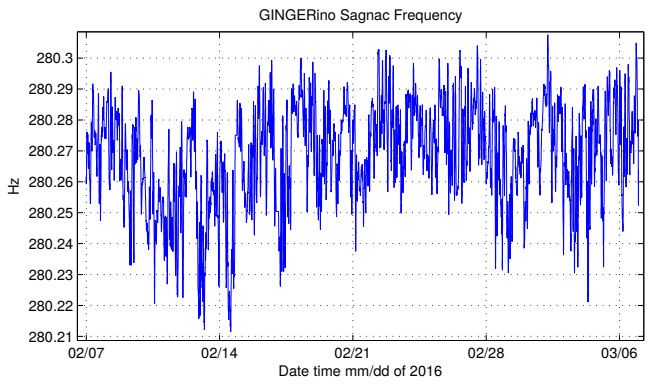

Fig. 6: Raw data time series of 29 days of continuous acquisition of the Earth's induced Sagnac frequency. The instrument duty cycle is approximately $100 \%$. Sampling interval of this time series is 1 sample per hour.

\section{CONCLUSION}

The present status of the experimental activity toward General Relativity tests with ring lasers has been discussed. Two prototypes GP2 and GINGERino are operating respectively at INFN Pisa and inside the LNGS deep underground laboratories. The GP2 control system is under study and the diagonal resonators length control has been demonstrated with a precision at the nanometric level. Next steps toward the demonstration of an enhanced stability Sagnac interferometer, controlled both in diagonals and perimeter, are ongoing. 


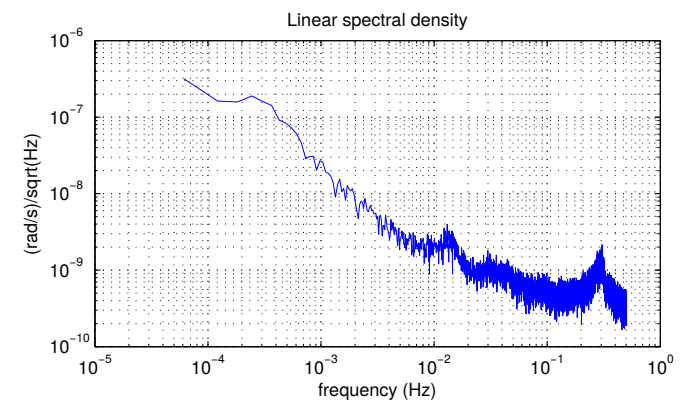

Fig. 7: Raw data linear spectral density of the rotational signal of GINGERino.

GINGERino is taking data in free running mode (without any active stabilization system up to now). The passive stability of the environment makes it possible to perform continuous acquisition of the Sagnac frequency on the monthly timescale. The present performances of GINGERino are limited by the large amount of backscattered light coming from the low quality set of mirrors. Large improvements are expected after the installation of a new set of mirrors and the implementation of the perimeter length stabilization.

\section{REFERENCES}

[1] K. U. Schreiber and J-P. R. Wells, Rev. Sci. Instrum. 84, (041101), (2013).

[2] F. Bosi et al., Phys. Rev. D 84, 1220022, (2011).

[3] https://web2.infn.it/GINGER

[4] http://arxiv.org/abs/1601.02874

[5] K. U. Schreiber et al., Phys. Rev. Lett., 107, 173904, (2011).

[6] D. Cuccato et al., Metrologia 51, 97107, (2014).

[7] R. Santagata et al., Class. Quantum Grav. 32, 055013, (2015).

[8] J.Belfi et al., Class. Quant. Grav. 31, 22500, (2014).

[9] J. Belfi et al., Appl. Phys. B, 106, 271-281, (2012). 